\title{
What's in a task? Complications in the study of the task-unrelated-thought (TUT) variety of mind wandering
}

${ }^{\mathrm{a}}$ Samuel Murray, ${ }^{\mathrm{b}}$ Kristina Krasich, ${ }^{\mathrm{c} J}$ onathan W. Schooler, and ${ }^{\mathrm{a}, \mathrm{b}}$ Paul Seli

aDepartment of Psychology and Neuroscience, Duke University, Durham, NC, USA

${ }^{\mathrm{b}}$ Center for Cognitive Neuroscience, Duke University, Durham, NC, USA

'Department of Psychological and Brain Sciences, University of California Santa Barbara, Santa

Barbara, CA, USA

\section{Author Note}

Correspondence may be directed to Samuel Murray, Department of Psychology and

Neuroscience, Duke University, 417 Chapel Dr., Durham, NC, 27708. Email:

samuel.f.murray@duke.edu. 


\begin{abstract}
In recent years, the number of studies examining mind wandering has increased considerably, and research on the topic has spread widely across various domains of psychological research. Athough the term "mind wandering" has been used to refer to various cognitive states, researchers typically operationalize mind wandering in terms of "task-unrelated thought" (TUT). Research on TUT has shed light on the various task features that require people's attention, and on the consequences of task inattention. Important methodological and conceptual complications do persist, however, in current investigations of TUT. As we argue, these complications may be dampening the development of a more nuanced scientific account of TUT. Here, we outline three of the more prominent methodological and conceptual complications in the literature on TUT, and we discuss potential directions for researchers to take as they move forward in their investigations of TUT.
\end{abstract}

Keywords: Task; Task-unrelated thought; TUT; Mind Wandering 


\section{Introduction}

\subsection{The Emergence of Scientific Interest in Mind Wandering}

Over the past decade, psychological science has come to appreciate the importance of the mind's tendency to engage in rich, internal thought that is unrelated to the here and now, or “mind wandering." Scientific investigation of mind wandering has truly burgeoned in recent years, with some describing this as the "era of the wandering mind" (Callard, Smallwood, Golchert, \& Margulies, 2013). Through 2006, there was a dearth of research examining mind wandering. In more recent years, however, there has been an enormous increase of interest in the topic, with 92 papers on mind wandering published already in 2019 (see Figure 1). 


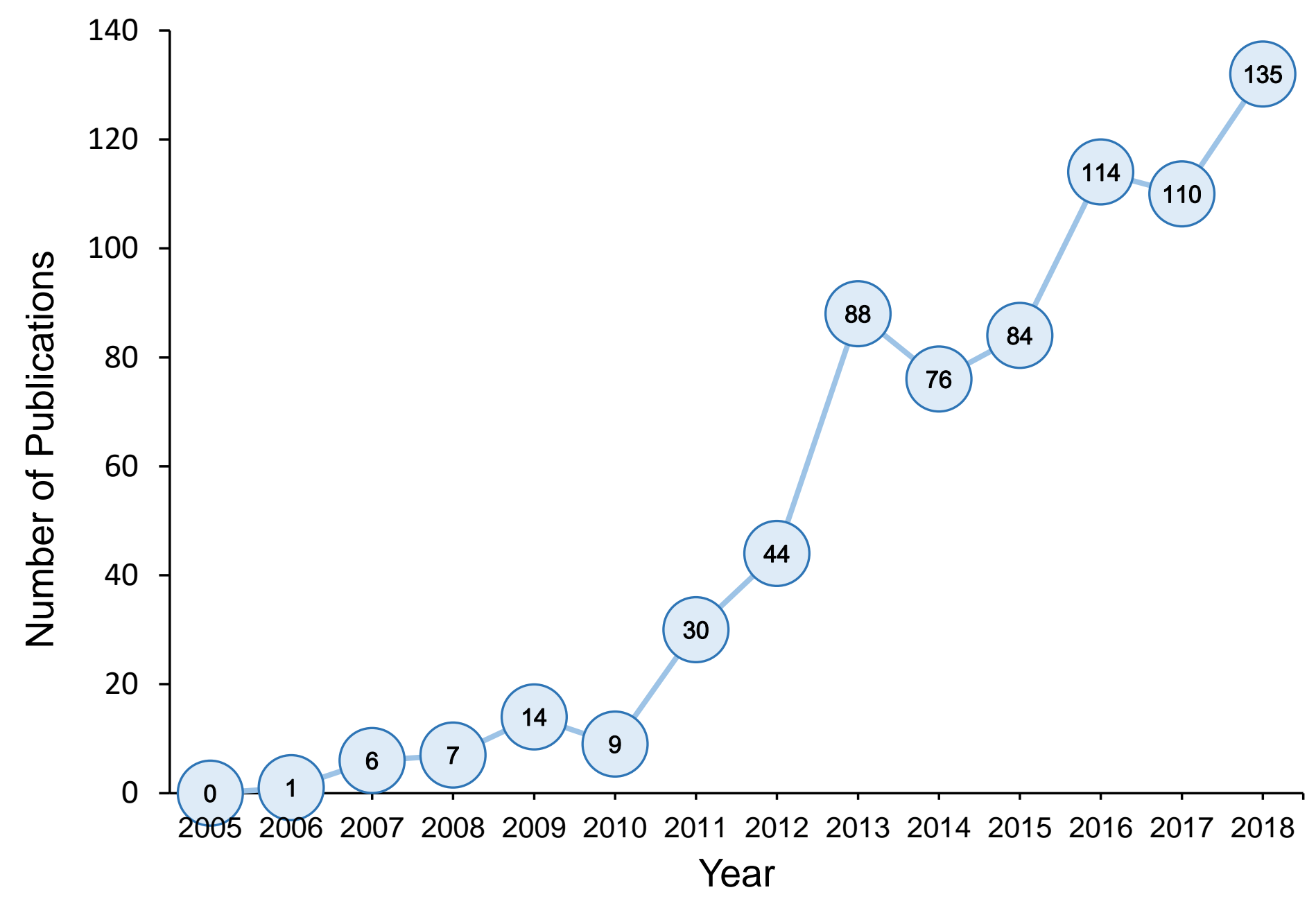

Figure 1. Changes in the number of publications focused on mind wandering from 2005 to 2018 . The data for this figure were obtained (on August 30, 2019) by conducting a search, via pubmed.org, for any articles that used the term "mind wandering" in the title/abstract of the article. 
The striking escalation in mind-wandering research over the last 10 years raises the question: what happened to make mind wandering such a popular topic? Several factors may have conspired to excite research on mind wandering (see Callard et al., 2013 for further discussion). Mind wandering occurs somewhat regularly, with conservative estimates stating that around $20 \%$ of waking hours are spent mind wandering (Seli, Beaty, et al., 2018; less conservative measures estimate that around $50 \%$ of waking hours are spent mind wandering, as in Kane et al., 2007 and Killingsworth \& Gilbert, 2010). Mind wandering has unique costs and benefits. It has been found to disrupt primary task performance and rivals intoxication and texting as a source of automobile accidents (Yanko \& Spalek, 2013). However, mind wandering is potentially beneficial and is connected with relief from boredom (Baird, Smallwood, \& Schooler, 2010), positive mood (Franklin et al., 2013), and attenuation of habituation (Mooneyham \& Schooler, 2016).

Two methodological trends also help to explain growing interest in mind wandering. First, evidence has been accumulating over the past ten years that documents the validity of selfreport measures in mind wandering research by demonstrating convergence between self-reports and a host of other indirect measures that could reasonably be expected to correspond with mind wandering. This includes behavioural measures such as performance (McVay \& Kane, 2009) and changes in response variability (Seli, Cheyne, \& Smilek, 2013), as well as physiological measures such as evoked response potentials (Smallwood, Beach, Schooler, \& Handy, 2008) and gaze behavior (Krasich et al., 2018). Second, the advent of experience-sampling methodologies (for reviews, see Csikszentmihalyi \& Larson, 1987; de Vries, Dijkman-Caes, \& Delespaul, 1990; Hurlburt, 1997) allowed researchers to sample the occurrence of mental events in everyday life. This enabled comparisons between mind wandering in the laboratory with mind wandering in 
daily life.

One critical factor that we focus on here was inspired by a widely cited review by

Smallwood \& Schooler (2006). Until this review, mental states that subsequently would be classified as mind wandering were characterized by a host of obscure and unfamiliar names (e.g., task-unrelated thought; stimulus-independent thought; mind pops, etc.). However, Smallwood \& Schooler (2006) argued for the value of using the single term 'mind wandering' as the umbrella term to describe the various phenomena, noting:

Perhaps part of the reason why mind wandering has escaped mainstream attention is that research addressing the issue has been framed in the context of a variety of disparate constructs... These various lines of research have all addressed the basic phenomenal characteristics of mind wandering, a shift of attention away from a primary task towards internal information, such as memories.... by referring to this phenomenon as mind wandering, a term familiar to the lay person, we hope to elevate the status of this research into mainstream psychological thinking (Smallwood \& Schooler, 2006, p. 946).

As can be seen in Figure 1, following this review, the field witnessed dramatic increases in both research on this general topic and the specific preference for the moniker of 'mind wandering' over other terms. It thus seems that Smallwood and Schooler were successful in spurring the field forward in the investigation of mind wandering by encouraging the use of that particular term.

\subsection{Challenges to Scientific Research on Mind Wandering}

Although the field appears to have been stimulated by the convergence of disparate research traditions under the rubric of mind wandering, this may not have been without some costs. One such cost becomes evident when considering the challenges of experimentally operationalizing the concept of 'mind wandering' — a step that is necessary when assessing mind wandering in laboratory settings and in daily life, and which is essential for theoretical progress. 
Due to differences between research traditions and research questions, these operationalizations varied substantially between studies, often picking out overlapping — though distinct — cognitive states. Despite this, it has become common practice for researchers to discuss results in terms of a generic concept of mind wandering without noting these subtle differences.

Some researchers have made the case against this practice and argued for more nuance in the study of mind wandering (see Seli, Risko, Smilek, \& Schacter, 2016 for review). A recent review from Seli, Kane, et al. (2018) provides a general argument that mind wandering is a heterogeneous construct with multiple, overlapping attributes that no single instance of mind wandering fully exemplifies, such as task-unrelatedness, stimulus independence, lack of guidance, intentionality, and others (see also Seli, Kane, Metzinger, et al., 2018). This creates a problem of generalizing across different empirical results from studies that adopt disparate operational definitions of 'mind wandering'. Grouping various empirical results may obscure important details and distinctions and, for this reason, hinder the emergence of a mature scientific account of mind wandering that is sensitive to the complex and often heterogeneous nature of the experience. In light of the different kinds of mind wandering, Seli, Kane, et al. (2018) suggested that, to avoid generalization problems, researchers should identify the kind of mind wandering they are studying. In line with the suggestions of Seli, Kane, et al. (2018), our aim was to consider, in isolation, one variety of mind wandering that has received considerable attention over the past decade or so ("task-unrelated thought") and to specifically focus on the current conceptual and theoretical challenges faced by researchers of task-unrelated thought (TUT).

\subsection{Task-Unrelated Thought}


To date, the most common approach taken by researchers has been to operationalize mind wandering as cognitive processing that is unrelated, in varying degrees, to some ongoing, externally oriented task (i.e., TUT). Indeed, as recently highlighted, of the peer-reviewed articles published in 2016 with "mind wandering" in the title, $94.5 \%$ referred to mind wandering as reflecting some form of TUT (Mills, Raffaelli, Irving, Stan, \& Christoff, 2018).

The adoption of a TUT conceptualization of mind wandering has been beneficial because investigations of TUT have allowed researchers to determine the various task features that require people's attention, as well as the (often-negative) consequences of failing to attend to ongoing tasks. For instance, research has examined TUT in the context of learning in educational settings (Wammes, Boucher, Seli, Cheyne, \& Smilek, 2013), reading (Feng, D’Mello, \& Graesser, 2013; Schad, Nuthmann, \& Engbert, 2012), and driving (Yanko \& Spalek, 2013; 2014), all of which are tasks during which TUT tends to have negative consequences for performance.

Although studies of TUT have shed light on the causes, consequences, and correlates of task inattention in a variety of important contexts, some notable methodological and conceptual complications exist in this current period of research on the topic. These complications can hinder the development of a more nuanced scientific account of TUT. Addressing these complications provides fertile grounds for future research on the topic. Here, we discuss three methodological and conceptual complications with the current scientific study of TUT and discuss potential directions for researchers to take moving forward.

\section{Three complications in studies of task-unrelated thought}

\subsection{When is a thought task-unrelated?}


One complicating factor in studies of TUT is that what constitutes a task is not clear. Consequently, it is not clear which thoughts ought to be classified as task-unrelated versus taskrelated. In most laboratory investigations of TUT, researchers have assigned to participants a putative "focal task," and thoughts that are not directly related to these researcher-imposed tasks have been classified as TUTs. Indeed, to date, one of the most common methods for indexing TUT in the laboratory has been to intermittently probe participants to report whether, just prior to the onset of a probe, they were "on task" (i.e., focused on the researcher-imposed task) or "mind wandering" (i.e., thinking about things unrelated to the researcher-imposed task; for a review, see Weinstein, 2018). Importantly, inherent in this practice is the assumption that a researcherimposed task corresponds to the participant's primary (focal) task; that is, researchers typically assume that participants fully intend to perform the researcher-imposed task.

Because the notion of task is central to the study of TUT, we want to introduce some conceptual machinery that distinguishes tasks from goals and where tasks fit into the broader tapestry of human agency. In our view, tasks are concrete routines enacted through a series of coordinated, congruent actions that facilitate achieving goals. Thus, performing a task requires goal pursuit. Maintaining an intended goal, however, does not require having a task immediately enacted. Goals reflect desires to perform activities with valuable termini (see Klinger, 2013), but goals can be ambiguous, wide-ranging, and enacted later in time. For example, goals can be as, diverse as 'learn a new language' or 'get in shape'. Other goals are a little more well-defined, such as 'finish writing the manuscript by next week'. Following our view, a goal (learn a new language) is achieved by performing a relevant task or multiple tasks (completing an online language course). 
Goals are always self-selected, even though adopting a goal might occur in virtue of deception or manipulation. Tasks, on the other hand, can be either self- or other-imposed. For example, one goal might consist in keeping one's job or being a good spouse. This might sometimes require performing a task because your boss or spouse tells you to do something. Performing self-selected or other-imposed tasks might require the same bodily movements (organizing a spreadsheet is the same no matter how you slice it), but the difference might reflect motivational and experiential differences in task performance.

What makes something a primary (or focal) task is that the individual intends to perform a specific task in the present to pursue a goal. A real life example might describe someone preparing dessert by baking a cake and making some frosting. Both of these, in turn, require the performance of specific subroutines (preheat the oven, beat eggs, mix dry ingredients, etc.). In a laboratory setting, researchers ask participants to complete specific tasks, and the hope is that the participant's goal is to follow the researcher's instructions and complete the task with care. Having the intention to complete the task generates further commitments to guide one's attention in ways that contribute to performing the task (see Figure 2). ${ }^{1}$ However, people can (and often do) have multiple overlapping goals that they are equally interested in realizing at any given time. People can then shift attention between different goals and their associated task sets. This alters the balance of the person's priorities, which in turn alters their attentional commitments. This is important because it shows that an individual can engage in thoughts that: (a) are unrelated to a current task but (b) contribute to performing a different task. In the laboratory, this might manifest as a shift of attention from the researcher-imposed task to some other (internally-

\footnotetext{
${ }^{1}$ The person's intention, however, only generates these commitments in virtue of some overarching goal to which the person is committed. Committing attention to an intended task, then, depends on an overarching commitment to a goal that the intention (if executed successfully) serves to advance (see Klinger, 2013).
} 
oriented) task that relates to a different goal. ${ }^{2}$ For example, a researcher might instruct a participant to perform a 1-back task, but the participant can (perhaps because of boredom or fatigue) decide or slip into thinking about what to buy later at the grocery store. This idea is described in detail in the goal theory of current concerns, which proposes that mind wandering occurs when engaging with internal goals and desires has a higher incentive value than attending to the external environment, and, therefore, mind wandering is rooted in competition between self-relevant, internal priorities and task-relevant, external priorities (Klinger, 1971, 2013, 2018;

Klinger \& Cox, 2011; Klinger et al., 2018).

\footnotetext{
${ }^{2}$ This does not mean that previous intentions are cancelled; rather, they occupy a diminished role in the person's hierarchy of priorities (which affects cognitive resource allocation). Thus, in the lab, a participant might shift focus to some other task while still maintaining enough attention to accurately respond to the researcher-imposed task. This is because participants can think about a task without focusing on it.
} 


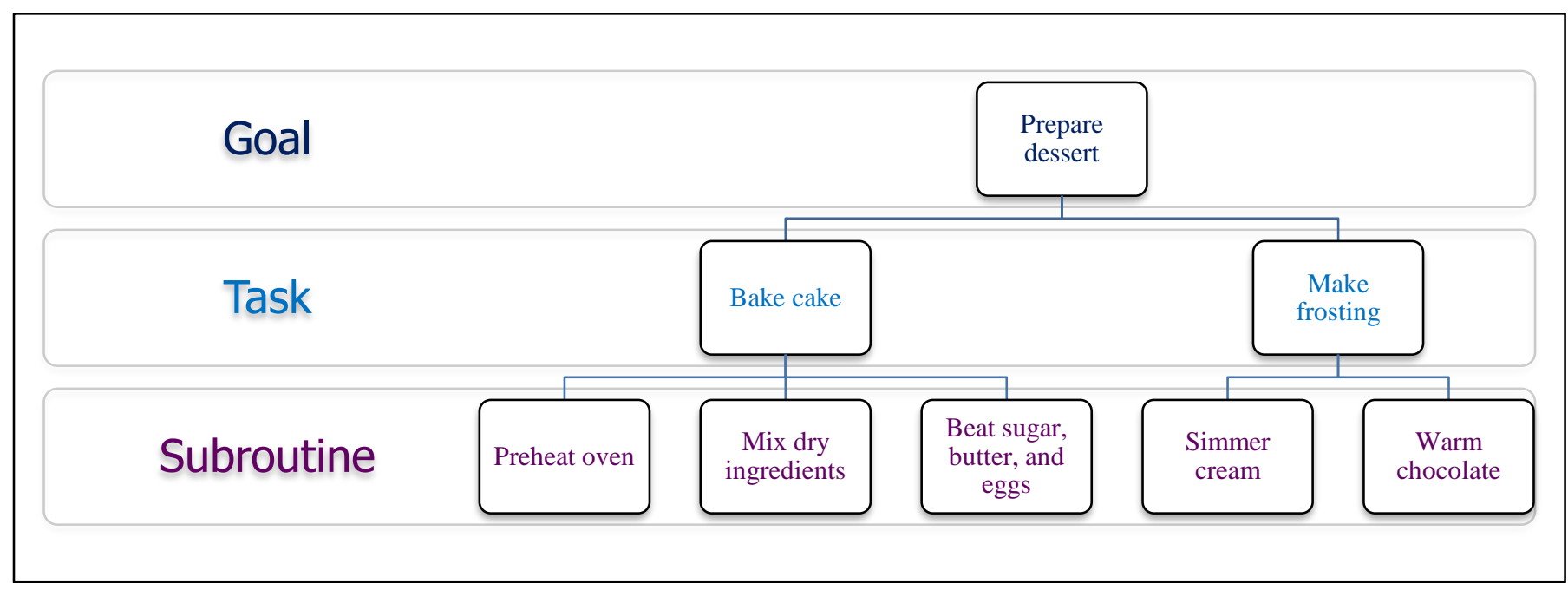

Figure 2. Structure of Activities. This figure depicts the relationship between goals, tasks, and subroutines. When someone intends to perform a task (baking a cake) or a subroutine (mixing dry ingredients) in order to achieve their goal (preparing dessert), they thereby adopt commitments to guide attention in ways that support performing these tasks and subroutines. For example, one must pay attention to the necessary amounts of ingredients, how well things should be mixed, etc. This figure also shows how someone could engage in TUT without thereby neglecting their goal. Someone could, for instance, mix dry ingredients while thinking of how best to make the frosting. This would be a case of TUT that was still related to the overall goal of making dessert. The differences across the hierarchy can be accounted for in terms of inherent structure. Goal are less structured than tasks, as there are many ways to prepare dessert without baking a cake. Tasks are less structured than subroutines, as one could bake a cake that does not require beating butter, sugar, and eggs. Finally, note that laboratory activities are typically much less complicated than this daily life example. A participant performing a vigilance task in lab has one goal (comply with the researcher), one task (the vigilance task), and presumably one subroutine (monitor for target stimuli, and produce a response when appropriate).

What the foregoing suggests is that there are various kinds of off-task thought that can occur in the context of task performance. One kind manifests task inattentiveness. The participant intends to perform the researcher-imposed task and so commits to attending to the task. But, for whatever reason, their attention gets pulled to something that doesn't contribute to task performance and is not related to executing the intention. However, a different kind of offtask thought manifests goal substitution. That is, the participant shifts to pursuing a different goal and their thoughts are related to executing this other intention. In this case, the participant adopts different attentional commitments (see Table 1). 
Table 1. Definitions and examples of Unintentional and Intentional TUT.

Variety of TUT Definition Example

Unintentional TUT
Thoughts that (a) are unrelated to a current task, and (b) occur despite the fact that the participant is committed to attending to the current task.
A researcher instructs a participant to complete a 1-back task. The participant is committed to attending to the 1back task, but inadvertently begins to think about thoughts unrelated to the 1-back task (e.g., grocery list).
Intentional TUT
Thoughts that (a) are unrelated to a current task, and (b) occur when the participant intends to think about something unrelated to the current task.
A researcher instructs a participant to complete a 1-back task. The participant is not committed to attending to the 1back task, and intentionally attempts to think about an unrelated activity (e.g., grocery list). 
Canonical examples of mind wandering construe it as disengaging from a current task and intentionally thinking about something else. This reflects one kind of intentional TUT, namely directed intentional TUT. This picks out thoughts that occur in situations wherein participants have intentionally shifted attention away from the current task and direct their thoughts to a different task. However, a second type of intentional TUT_-undirected intentional TUT_-includes thoughts that occur in situations wherein participants have intentionally disengaged from the current task but have not committed to direct their attention to any specific thing. In this case, the participant intentionally allows his mind to wander, drifting between different internally generated contents without any specific focus (see Table 2).

Note that the original distinction between intentional and unintentional TUT fails to capture the distinction between directed and undirected TUT. For one, undirected TUT, though intentionally initiated, may have internal dynamics that are more closely aligned with unintentional TUT than with directed TUT. Further, undirected TUT manifests task inattentiveness, whereas directed TUT might not (more on this later). Thus, researchers cannot assume that intentional TUT manifests task attentiveness given the differences between directed and undirected TUT. 
Table 2. Definitions and examples of various Intentional TUTs

Variety of Intentional TUT Definition $\quad$ Example

Directed Intentional TUT
Thoughts that are unrelated to a current task and occur when the participant commits to attending to another task.
A researcher instructs a participant to complete a 1-back task. The participant is not committed to attending to the 1back task, and intentionally attempts to think about an unrelated activity (e.g., making a grocery list).
Undirected Intentional TUT
Thoughts that are unrelated to a current task and occur when one's thoughts drift between different contents without committing to focus on any specific content.
A researcher instructs a participant to complete a 1-back task. The participant is not committed to attending to the 1back task, and intentionally lets his mind wander without directing his focus to a given activity. 
As stated earlier, directed intentional TUT might manifest task inattentiveness, but it might not (see Table 3). Successfully directed TUT occurs when a participant commits to attending to some other task and successfully maintains her attention on this self-selected task. ${ }^{3}$ Unsuccessfully directed TUT (as the name suggests) occurs when a participant switches tasks and fails to maintain attention on this other task. ${ }^{4}$

Our distinctions are not drawn in ways that reflect potential differences or similarities in either content or dynamics. This way of carving the terrain might seem odd for two reasons. First, distinct forms of TUT (on our scheme) might overlap in content or dynamics, thus rendering a distinction inapt or misleading. Second, the dynamics of TUT seem more relevant to understanding the psychological mechanisms and component processes that support TUT. Hence, distinctions that are insensitive to the dynamics of TUT do not help advance the scientific study of TUT.

Research on TUT relies on self-report wherein features of TUT are measured at single time points using thought probes administered throughout an experiment. Using several observations, researchers can interpolate to stitch together an account of a participant's overall TUT episode. These probes, however, are limited to studying features like intentionality, directedness, and motivation. That is why our taxonomy carves TUT according to these various dimensions. Methodological advances might generate opportunities to draw the taxonomy differently, but we currently do not have the methodological resources to do this.

\footnotetext{
${ }^{3}$ Interestingly, this variety of "TUT" is akin to being "on task" or "goal directed" because the participant is actively engaged with her other task. However, traditionally, such thoughts have been classified by researchers as failures of executive control.

${ }^{4}$ This variety of TUT is akin to a failure in cognitive control or a failure to maintain attention on the self-imposed task.
} 
Additionally, we believe that our taxonomy can help to generate new hypotheses and predictions about the mechanisms and component processes of TUT. While our categories may not shed light on components or mechanisms of TUT, there is no way to know this without first proposing distinctions and then testing for their empirical adequacy. By proposing a set of plausible behavioral categories, we can generate further hypotheses about: (a) how to differentiate forms of TUT, and (b) which mechanisms may support differential forms of TUT. Without these behavioral categories, it is difficult to determine how we would go about constructing mechanistic hypotheses of TUT. 
Table 3. Definitions and examples of various Directed TUTs

Variety of Directed TUT Definition $\quad$ Example

Successful Directed TUT
Thoughts that (a) are unrelated to a current task, (b) occur when the participant commits to attending to some other task, and (c) are successfully maintained on this other task.
A researcher instructs a participant to complete a 1-back task. The participant is not committed to attending to the 1back task, and deliberately attempts to think about an unrelated task (e.g., making a grocery list). The participant successfully allocates her attention to the task of making the grocery list.
Unsuccessful Directed TUT
Thoughts that (a) are unrelated to a current task, (b) occur when the participant commits to attending to another task, and (c) are inadvertently directed away from this other task.
A researcher instructs a participant to complete a 1-back task. The participant is not committed to attending to the 1back task, and deliberately attempts to think about an unrelated activity (e.g., making a grocery list). However, the participant unsuccessfully allocates her attention to the self-selected activity of making the grocery list, and instead thinks unrelated thoughts. 
We have argued for distinctions between various kinds of off-task thought, though only some of these seem to count as genuine instances of task-inattentiveness; namely, unintentional TUT, undirected TUT, and unsuccessfully directed TUT (see Figure 3). Other varieties of TUT (e.g., successfully directed TUT) do not count as instances of task-inattentiveness because in these situations, participants have committed to attend to some task and do so successfully. The illusion of task inattentiveness arises only if we assume that a researcher-imposed task always constitutes the participant's focal task.

Although it is plausible that participants engage in all three varieties of TUT in the laboratory, there is currently no vocabulary or methodology with which to distinguish among these different types of experience. Recognizing these distinctions is critical because these three varieties of TUT likely reflect different types of cognitive processes and, thus, conflating them is problematic. Directed TUT, for example, may largely be driven by lack of motivation to perform the researcher-imposed task, whereas unintentional TUT may derive from control failures. Moreover, unintentional TUT and unsuccessfully directed TUT may be more likely than successfully directed TUT to be attributable to failures of executive control (see Robison \& Unsworth, 2018; McVay \& Kane, 2010). ${ }^{5}$

\footnotetext{
${ }^{5}$ Even though unintentional TUT and unsuccessfully directed TUT typically reflect failures of executive control, these failures need not be interpreted as maladaptive. Often, the interfering thoughts will be related to some other goal or personal concern even if they are not directly related to the focal task (cf. Klinger et al., 2018).
} 


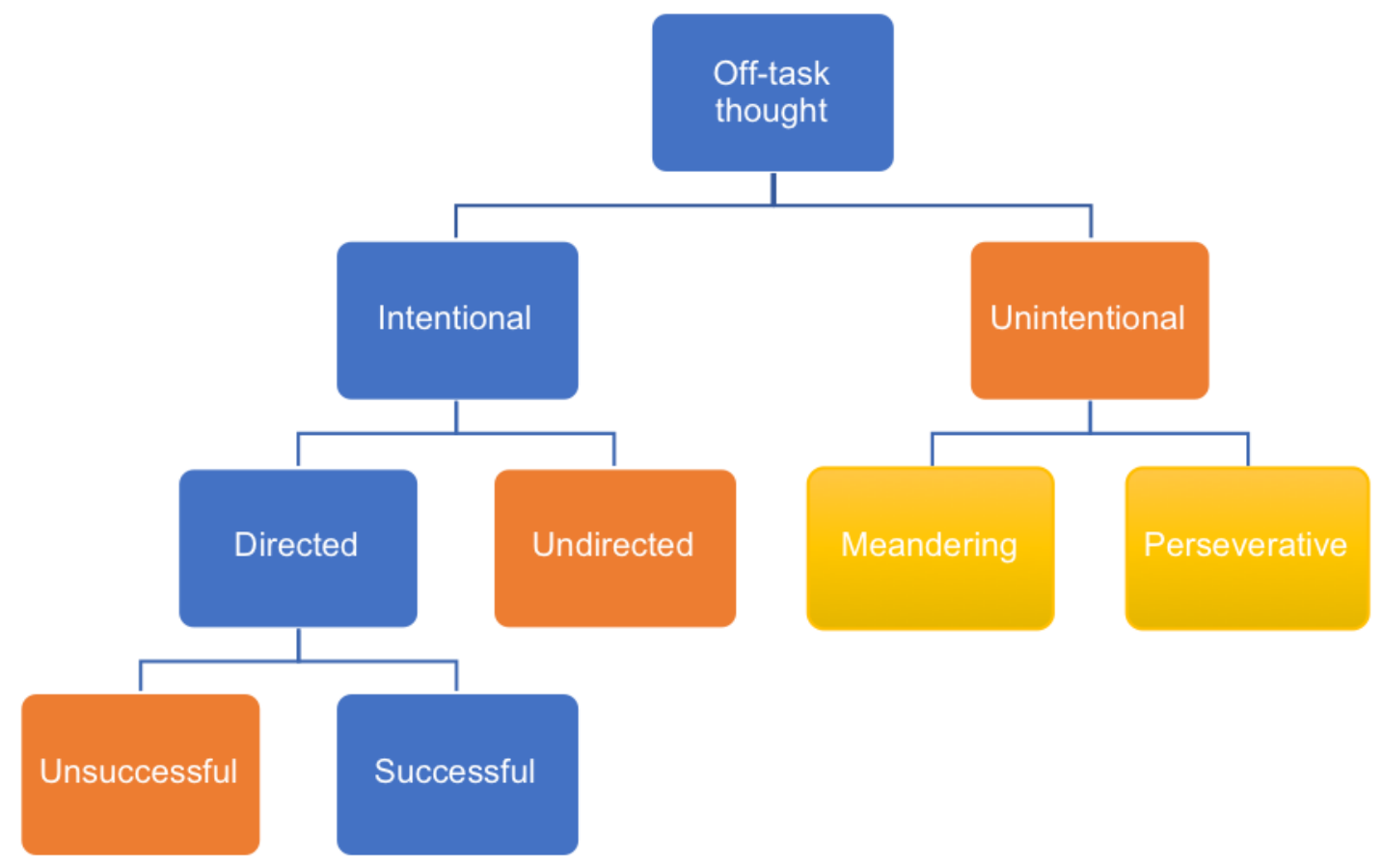

Figure 3. Taxonomy of TUT. This classification is based on the distinctions mentioned above. Highlighted in orange are the kinds of TUT that exhibit different forms of task inattentiveness. However, these different forms of TUT may reflect dissociable cognitive processes and should not automatically be lumped together. Unintentional meandering and perseverative TUT are displayed in yellow to highlight the fact that these forms of thought may or may not exhibit task inattentiveness, as both may momentarily include task-related contents. Highlighted in blue are different forms of intentional TUT that do not reflect task inattentiveness despite potentially constituting off-task thought. 
This latter point is particularly noteworthy since one of the dominant theoretical accounts of mind wandering, the Executive Control Failures $\times$ Concerns account (McVay \& Kane, 2010; see also Klinger, 1978-79 and Klinger \& Cox, 1987-88), posits that TUTs reflect failures to “defend primary-task performance against interference from ... thoughts" (McVay \& Kane, 2010: 195). On this account, TUTs occur when an individual fails to allocate attention properly. Although a common practice has been to assume that all thoughts that are unrelated to a researcher-imposed task must reflect such control failures (e.g., Blanchard et al., 2014; Carciofo et al., 2014; Kane \& McVay, 2012; McVay \& Kane, 2010; Qu et al., 2015; Rummel \& Boywitt, 2014), successfully directed TUT would, if anything, reflect a success of cognitive control, since the participant would have effectively allocated his attention to a task he committed to completing. Thus, without distinguishing these different varieties of TUT, researchers who are interested in investigations of failures of cognitive control may unwittingly conflate actual failures of control with successful exercises of control (cf. Thomson, Besner, \& Smilek, 2015).

For this reason, we believe that our distinctions are important for developing more nuanced theoretical accounts of TUT. Current accounts make assumptions about the kinds of thoughts participants are reporting that, according to our scheme, are incorrect (e.g., TUT always reflect unintentional thought, TUT reflect failures of control, or that intentional TUT must reflect a success of cognitive control).

Our proposed distinctions are not pulled from thin air. Recent work on the intentionality of TUT establishes important precedents for our proposed taxonomy (see Seli, Risko, \& Smilek, 2016b; Seli, Risko, Smilek, \& Schacter, 2016). This research supports a distinction between unintentional and intentional forms of TUT, which informs the first distinction listed in Figure 3. Importantly, however, the intentional/unintentional distinction only captures some of the nuances 
of TUT. Indeed, it would appear that unintentional TUT and unsuccessfully directed TUT are both typical consequences of a failure to allocate one's attentional resources toward an activity that one has committed to performing (that is, a failure of executive control). The problem, however, is that the intentionality distinction does not capture this. The following examples make this clear:

1. A participant is assigned a sustained-attention task, but instead of focusing on the task, he intentionally begins constructing a grocery list and successfully maintains his attention on this activity (until now, these thoughts would be classified as intentional TUT, but, according to our proposed terminology, they would be classified as successfully directed TUT).

2. A participant is assigned a sustained-attention task, but instead of focusing on the task, she intentionally begins constructing a grocery list and fails to maintain her attention on this activity (until now, these thoughts would likewise be classified as intentional TUT, but by our terminology, they would be classified as unsuccessfully directed TUT). ${ }^{6}$

Armed only with the distinction between intentional and unintentional TUT, researchers would lump thoughts from scenario (1) with scenario (2). But, there appear to be real (empirically tractable) differences between the processes underlying these two streams of thought. So, although research focused on distinguishing between intentional and unintentional

\footnotetext{
${ }^{6}$ In this example, it is admittedly unclear how a participant would respond to the traditional thought probe asking her to indicate whether she was (a) on task, (b) intentionally mind wandering, or (c) unintentionally mind wandering. Certainly, the participant would not select the "on-task" option and, moreover, it seems unlikely that the participant would select the unintentional-mind-wandering option (i.e., "Thinking about task-unrelated thoughts despite my best intentions to focus on the task.") Presumably she would respond with the intentional-mindwandering option (i.e., "deliberately thinking about something other than the task"), although this option does not quite map onto her experience, either, since she was not successfully thinking about the self-imposed task.
} 
TUT has been fruitful, as we move forward in our investigations of TUT, we will require more precise terminology and correspondingly appropriate methodologies to allow us to deconfound these three varieties of TUT.

This consideration could be accommodated in the laboratory by requiring participants to, upon presentation of each thought probe, indicate whether they were focused on completing the researcher-imposed task or whether they were focused on something else (this distinguishes intentional from unintentional TUT). If the participant is focused on the researcher-imposed task, a follow-up about whether the person was thinking about the task or not would differentiate unintentional TUT from on-task thought. If the participant is focused on something else, then it needs to be determined whether the participant was thinking about something specific or just letting his thoughts drift. Drifting thoughts count as undirected intentional TUT, whereas specific thoughts indicate directed TUT. Finally, one could ask participants whether they successfully maintained focus or whether they were distracted. The former counts as successfully directed TUT whereas the latter counts as unsuccessfully directed TUT. Figure 4 represents a series of sample thought probes that correspond to the taxonomy outlined in Figure 3. 


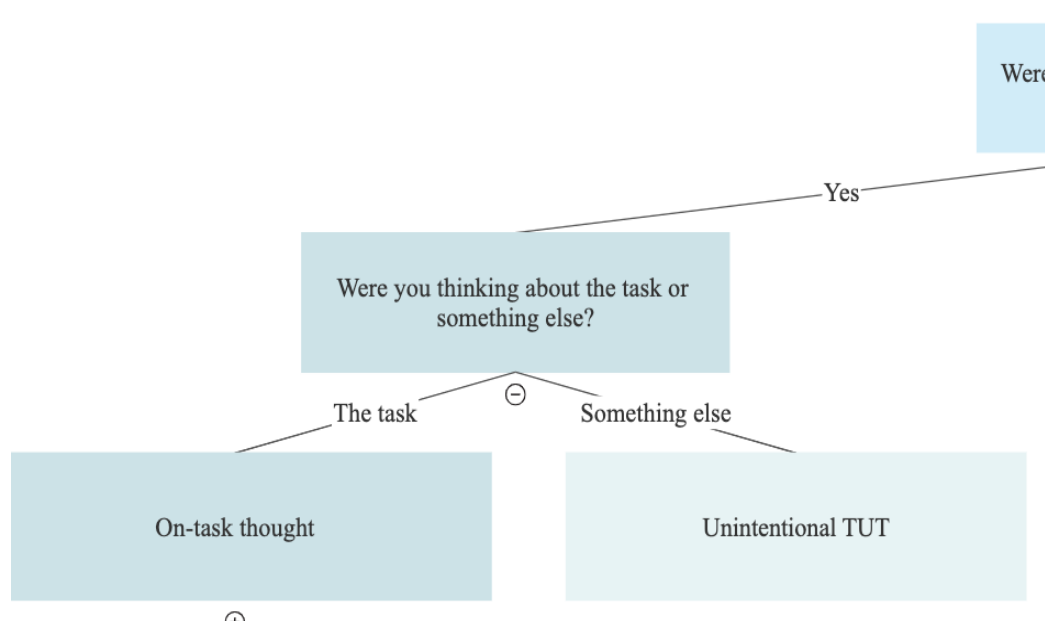

$\oplus$

re you actively trying to focus on the task
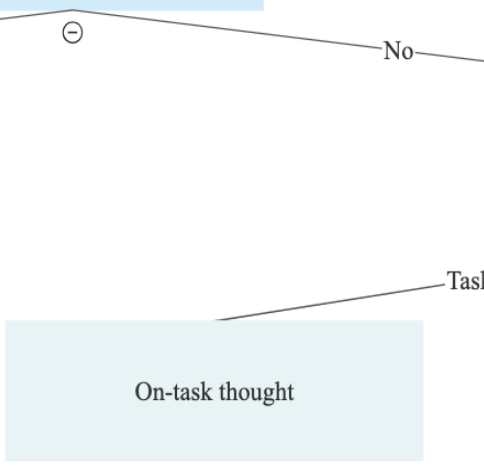

Were you thinking about the task without

focusing on it, thinking about something

specific or just letting your thoughts drift?

Task Drift

Specific (Directed Intentional TUT)

Undirected Intentional TUT

Did you maintain focus or did you ge distracted?

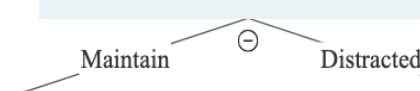

Successfully directed TUT

Figure 4. Sample series of thought probes that distinguish various kinds of TUT. 


\section{Daily-life TUT differs from laboratory TUT}

The study of TUT extends beyond the laboratory, where researchers are interested in the frequency and content of TUT in daily life. There are several methodological concerns with these daily life studies, however. First, whereas researchers have traditionally been interested in thoughts unrelated to an other-imposed task, to date, daily-life studies of TUT rarely include such tasks, or rarely attempt to determine whether people are performing self-selected or otherimposed activities. Second, researchers have tended to assume that in daily life, as in the laboratory, participants are always completing some sort of focal task-although clearly not a researcher-imposed task. However, there is currently an absence of support for this critical assumption, and introspection suggests that the assumption is likely incorrect. We outline two complications with daily-life studies of TUT based on these considerations.

\subsection{Some daily life tasks differ from researcher-imposed tasks}

Researchers tend to treat daily-life TUTs as being equivalent (or at least, directly comparable) to laboratory-based TUTs (e.g., Kane et al., 2017; Song \& Wang, 2012). While there are no researcher-imposed activities in daily life, there are other-imposed activities (e.g., your spouse asks you to wash the dishes or your boss asks you to fill out some additional paperwork). This suggests that the taxonomy of TUT outlined in Figure 3 also applies to TUT in daily life. While the distinctions depicted in Figure 3 certainly apply in many cases, their application elides a crucial difference between some daily-life tasks and laboratory tasks.

The performance of daily life tasks often does not require sustained attention for adequate performance. People can strategically allocate attention to compensate for waning motivation or fatigue. Suppose someone is writing a manuscript. They might write for 30 minutes and then 
take a 10 minute break to get coffee or take a walk. But, the "30 minutes on/10 minutes off" approach might reflect strategic task performance. From the individual's perspective, it might still be the case that she is writing her manuscript even while taking her 10 minute break. In this situation, the application of TUT is unclear. Because not all task performance requires sustained attention, individuals can still be performing a task without thinking about it in the moment. Current daily life methods presume that people are either engaged in a focal task or doing nothing. But the previous example show that daily life is more fluid.

The vast majority of tasks employed in laboratory-based studies of TUT do not exhibit these fluid characteristics (but see Seli, Carriere, et al., 2018). Laboratory tasks employed in the study of TUT tend to require rapid perceptual discriminations of transient signals in order to make accurate behavioural responses. Without a high degree of attentiveness, people are liable to make inaccurate responses (Seli, Carriere, et al., 2014). These tasks also tend to be quite boring and are very different from daily life activities (knitting, baking, writing, etc.). This does not imply that laboratory tasks are inherently more attentionally demanding than daily life activities. While researchers can manipulate attentional load, they often fail to manipulate these loads outside of a minimal range that hovers toward maximally demanding.

This suggests a few methodological problems with directly comparing laboratory and daily life studies of TUT. First, different kinds of tasks generate different classifications of thoughts associated with such tasks. For example, strategically engaging in a task implies that not every thought unrelated to such activities qualifies as TUT (i.e., it is not obvious that every thought a person has on a 10-minute break from her morning writing counts as TUT). In fact, it is not clear whether people can experience TUTs in moments of taking a planned, limited break from a task. Second, laboratory-based task performance is typically sensitive to even small degrees of 
task-inattentiveness (Schad et al., 2012; Seli, Carriere, et al., 2014), whereas many of the activities in daily life tolerate moderate levels of inattentiveness. Someone might, for example, hear a melody in their head while washing the dishes. This would qualify as TUT in a laboratorybased study (assuming the participant hadn't wilfully shifted his attention to "singing" the song internally, thereby making such thoughts directed intentional TUT), but because it does not affect task performance, the individual may not judge that he is being inattentive (or report that he is mind wandering).

The issue here is not that daily-life tasks and laboratory-based tasks are fundamentally different; rather, the issue is that current daily life methodologies are not discriminative enough to capture the various ways that people structure their activities in daily life. Laboratory-based tasks tend not to permit strategic engagement. Another way to put the difference is that thoughts related (or unrelated) to strategic tasks are not readily classifiable as on-task or off-task. Thus, how to classify thoughts related (or unrelated) to strategically engaged tasks is an important question for future research to tackle.

2.2 Task-free and task-ambiguous scenarios in daily life

Another problem with daily-life investigations of TUT is that researchers have tended to assume that, in their daily lives, people are always engaged in a task away from which their thoughts can wander to unrelated content (e.g., Kane et al., 2007; 2017; Killingsworth \& Gilbert, 2010; McVay, Kane, \& Kwapil, 2009; Song \& Wang, 2012). One concern with this assumption, as noted above, is that some tasks are temporally fluid to a degree that people can fail to fully attend to those tasks without those thoughts qualifying as TUT. Another concern is that daily life consists of task-ambiguous or task-free scenarios. For instance, while sitting on a bus or relaxing 
on the beach, one could argue that the individual is not completing a focal task, and, consequently, TUT is precluded in such contexts. However, sitting on the bus might be a component of a larger plan such as traveling to the grocery store, thus making the scenario taskambiguous. The ambiguity arises when it is unclear whether the individual views sitting on the bus as being related to the task of getting groceries. Moreover, relaxing on the beach is, arguably, a task-free scenario (unless we construe tasks so broadly so as to include activities such as relaxing on the beach, in which case, it is not clear what is not a task). Does this mean that all thoughts in such scenarios qualify as TUT, or does it mean that none do? Whatever the answer, it is clear that daily life is not simply a series of focal tasks, which in turn raises some important issues surrounding the methodological approaches currently adopted in daily-life studies of TUT.

Consider that many daily life studies of mind wandering use prompts that presume people are always performing a task (see Table 4). Most prompts ask whether one's current thoughts are directed at whatever one is currently doing. This might seem innocuous, but the prompt is ambiguous. On one reading, the prompt asks whether one's thoughts are focused on a current task (Ostojic-Aitkens, Brooker, \& Miller, 2019 make this explicit by using measures of on-task thought; see Table 4). On a different reading, the prompt asks the more general question of whether one's thought is directly related to a current activity. But, as already discussed, some activities make this question hard to interpret. This is because not all tasks impose consistently high attentional demands on a person.

Admittedly, Kane and colleagues (2017) acknowledge this possibility in their instructions to participants. Even though their examples of mind wandering all describe not thinking about a focal task, they describe situations where people are doing things that do not require any thought, like sitting on the couch or riding the bus. They label these as "doing-nothing occasions" and 
state that these are pretty rare. Participants are instructed to label all tasks in these situations as mind wandering. Our argument highlights a problem with this methodology. The presumption is that people are either performing focal tasks or doing nothing. But we've highlighted a third category, fluid tasks, that covers tasks that do not require full attentiveness for successful performance and have vague temporal parameters. The frequency of such tasks is unclear, in part because researchers have not designed methods to study these tasks.

This survey of daily life studies informs two lessons. Some situations are task-free or taskambiguous. Task-oriented definitions of mind wandering do not clearly preserve these distinctions, but many daily life studies continue to use task-oriented definitions of mind wandering (or prompts that could be interpreted as task-oriented). Second, some tasks can be strategically engaged. Current experience sampling methodologies do not have any way for participants to clearly communicate whether they are performing a temporally fluid task, thereby opening the possibility of confounds. Song \& Wang (2012) included a task question in their survey ("At the time of the signal, I was performing a task"). But the structure of the questionnaire presumes that mind wandering is just TUT. That is, if people indicate that their thoughts had not wandered off at the time of the signal, they were instructed to move directly to the task question. No results were reported for people that answered no to the off-task thought question and the task performance question. Thus, the structure of the questionnaire does not allow people to indicate the performance of fluid tasks.

A different structure, however, might be useful. Attention should be paid to two conditions: (a) people who respond 'Yes' to the off-task thought question and 'No' to the task question, and; (b) people who respond 'No' to the off-task thought question and 'No' to the task question. These situations reflect areas where researchers can draw out more nuanced 
information about the relationship between task performance and mind wandering. Further, we think that the instructions provided by Kane and colleagues about different task scenarios is highly useful. We are suggesting that more nuance be added to distinguish categories that map onto more nuanced dimensions of everyday experience.

Finally, we note that some daily-life studies are better than others. While we have focused on the work of Kane and colleagues, we commend their efforts to instruct participants on how to identify tasks and how to respond in potentially ambiguous scenarios. But other research does not reflect this level of nuance. As shown in Table 4, many daily-life studies implicitly study TUT and employ measurements of TUT without being sensitive to the complications raised here. Hence, we believe that the study of TUT in daily life would benefit from incorporating the suggestions we offer here. 


\begin{tabular}{|c|c|c|}
\hline Study & Prompt & Measure \\
\hline $\begin{array}{l}\text { Ostojic-Aitkens, Brooker, \& } \\
\text { Miller (2019) }\end{array}$ & $\begin{array}{l}\text { "Are you thinking about } \\
\text { something other than what } \\
\text { you're currently doing?" }\end{array}$ & $\begin{array}{l}\text { 5-point scale ("Completely on } \\
\text { task" to "Completely on } \\
\text { unrelated concerns") }\end{array}$ \\
\hline $\begin{array}{l}\text { Seli, Beaty, Cheyne, Smilek, } \\
\text { Oakman, \& Schacter (2018) }\end{array}$ & $\begin{array}{l}\text { (1) "At the time of the beep, } \\
\text { my mind had wandered to } \\
\text { something other than what I } \\
\text { was doing." } \\
\text { (2) "At the time of the beep, } \\
\text { my mind was..." }\end{array}$ & $\begin{array}{l}\text { (1) Dichotomous } \\
\text { (2) 4-point scale ("Completely } \\
\text { focused on what I was doing" } \\
\text { to "Mostly focused on } \\
\text { something other than what I } \\
\text { was doing") }\end{array}$ \\
\hline $\begin{array}{l}\text { Kane, Gross, Chun, } \\
\text { Smeekens, Meier, Silvia, \& } \\
\text { Kwapil (2017) }\end{array}$ & $\begin{array}{l}\text { "At the time of the beep, my } \\
\text { mind had wandered to } \\
\text { something other than what I } \\
\text { was doing." }\end{array}$ & $\begin{array}{l}7 \text {-point scale }(1=\text { not at all, } 4 \\
=\text { moderately, } 7=\text { very much })\end{array}$ \\
\hline $\begin{array}{l}\text { Franklin, Mrazek, Anderson, } \\
\text { Smallwood, Kingstone, and } \\
\text { Schooler (2013) }\end{array}$ & "Are you off-task?" & Dichotomous \\
\hline Song \& Wang (2012) & $\begin{array}{l}\text { "At the time of the beep, my } \\
\text { mind had wandered to } \\
\text { something other than what I } \\
\text { was doing." }\end{array}$ & $\begin{array}{l}5 \text {-point scale }(1=\text { not at all, } 5 \\
=\text { very much })\end{array}$ \\
\hline $\begin{array}{l}\text { Killingsworth \& Gilbert } \\
\text { (2010) }\end{array}$ & $\begin{array}{l}\text { "Are you thinking about } \\
\text { something other than what } \\
\text { you're currently doing?" }\end{array}$ & $\begin{array}{l}4 \text { items (No, Yes-pleasant, } \\
\text { Yes-neutral, Yes- } \\
\text { unpleasant) }\end{array}$ \\
\hline $\begin{array}{l}\text { McVay, Kane, \& Kwapil } \\
\text { (2009) }\end{array}$ & $\begin{array}{l}\text { First item asks whether } \\
\text { participant's current thoughts } \\
\text { had wandered from their } \\
\text { activity. }\end{array}$ & Dichotomous (Yes, No) \\
\hline $\begin{array}{l}\text { Kane, Brown, McVay, Silvia, } \\
\text { Myin-Germeys, \& Kwapil } \\
\text { (2007) }\end{array}$ & $\begin{array}{l}\text { "At the time of the beep, my } \\
\text { mind had wandered to } \\
\text { something other than what I } \\
\text { was doing." }\end{array}$ & Dichotomous (Yes, No) \\
\hline
\end{tabular}

Prompts and measures used in daily life studies of mind wandering 
Of course, research will be needed to identify a suitable methodology to achieve this goal, and we do not intend to propose or endorse any specific methodology. Instead, we raise this as a key issue that researchers of TUT would do well to consider if they are interested in examining dailylife TUT.

2.3 The consequences of inattentiveness to a given task

One goal of daily-life TUT research is to understand the various practical consequences of task inattention. For instance, research has examined the consequences of TUT in the classroom (Wammes, Seli, et al., 2016), while driving (e.g., Yanko \& Spalek, 2013), while reading (Feng et al., 2013), and so forth.

The kinds of tasks that structure daily life, as mentioned previously, are heterogeneous. The parameters of other tasks are ambiguous. Some tasks are performed intermittently. These various kinds of tasks are sensitive to differential ranges of task inattentiveness, where some tasks can be reliably completed without devoting full attention to performing those tasks (Thomson, Besner, \& Smilek, 2015). So, someone may be appropriately attentive to a given task without being fully attentive. Some daily-life TUT measures elide this distinction by measuring task attentiveness dichotomously. For example, Yanko \& Spalek (2013) measured mind wandering during a simulated driving task by asking whether participants were focused on the task or focused on something else in the ten second interval prior to an auditory thought probe. Similarly, Killingsworth \& Gilbert (2010) measured mind wandering by asking whether participants were “...thinking about something other than what [they are] currently doing?” This treats task attentiveness dichotomously, but the structure of daily life tasks means task attentiveness is graded. Some tasks require degrees of attentiveness that fall short of being fully 
attentive. Our methodological criticisms, then, suggest that researchers interested in the consequences of daily life TUT should consider using graded measures of task inattentiveness (see Seli, Beaty, et al., 2018 for further discussion). This recommendation applies only to research of daily life TUT, however. Indeed, recent evidence (Smith, Murray, Schooler, \& Seli, in preparation) suggests that continuous and dichotomous measures of in-lab TUT are equally predictive of errors on the 1-back task and the metronome response task (MRT: Seli, Cheyne, \& Smilek, 2013). Thus, laboratory-based studies of TUT can draw on continuous or dichotomous measures. Because dichotomous measures provide the appropriate structure for multiple classifications, there remains room for using some dichotomous measures of TUT.

\section{The assumption that measures of mind wandering or daydreaming apply to TUT}

One final complication with investigations of TUT is that researchers have often assumed that measures of TUT generalize to other kinds of mind wandering and, conversely, that measures of mind wandering and daydreaming also apply to TUT. The underlying assumption is that it is permissible to equate daydreaming with TUT. This ignores the fact that some scenarios are task-ambiguous or task-free, as mentioned above, and that some mind wandering and daydreaming could occur in the absence of TUT (Seli, Kane, et al., 2018). To return to an earlier example, neither sitting on a bus nor relaxing on the beach requires that the individual be currently performing a task, which thereby precludes TUT in such contexts. However, people in these situations might still mind-wander or daydream. The assumption that mind wandering implies TUT or that TUT measures generalize to all other forms of mind wandering, however, rules out this possibility. It seems clear that mind wandering is possible in task-ambiguous or 
task-free scenarios, which indicates that there is no simple inference from mind wandering to TUT.

Without acknowledging the possibility of mind wandering in the absence of TUT, researchers sometimes integrate information drawn from measures of TUT with information drawn from measures of other kinds of mind wandering (e.g., daydreaming or intentional mind wandering). For example, researchers might combine information about rates of TUT with the claim that mind wandering is associated with creativity without acknowledging the possibility that these measures are associated with different kinds of mind wandering. Given the possibility of mind wandering in the absence of TUT, researchers should be cautious when drawing general conclusions about mind wandering from information about TUT (see Seli, Kane, et al., 2018).

Similar problems arise for the inference from measures of daydreaming to measures of TUT. For example, it is common for researchers to use the Imaginal Processes Inventory (IPI; Singer \& Antrobus, 1972)—a retrospective self-report questionnaire assessing “daydreaming”as a proxy for TUT (e.g., Baird et al., 2012; Birnie et al., 2015; Qin et al., 2011; Shrimpton et al., 2017). Importantly, the daydreaming-related IPI questions (e.g., "Whenever I have time on my hands, I daydream," or "I lose myself in active daydreaming") do not require a focal task for "daydreaming" to be reported, so from the perspective of investigations of TUT, it is unclear whether responses to the IPI reflect TUT, task-free thoughts (which would, by definition, not qualify as TUT), or a combination of the two. One reason to be sceptical of the connection between daydreaming and TUT is that the properties of the content are different. Daydreams have somewhat explicit content and more narrative structure relative to TUT (Mason et al., 2007; Stawarczyk et al., 2012). These differences in content suggest that daydreams are a distinct kind of mental episode from TUT. It is entirely possible that daydreaming, like mind wandering, is a 
heterogeneous concept that also suffers from terminological vagueness (Zedelius \& Schooler, 2015). A recent theory of mind wandering, the Family Resemblances view, explicitly acknowledges that mind wandering is a multidimensional concept that cannot be classically defined (see Seli et al., 2018). If daydreaming and mind wandering are both heterogeneous concepts encompassing a wide range of different kinds of experiences, then there is certainly no straightforward generalization from TUT to daydreaming or from daydreaming to TUT.

Relatedly, these complications in comparing daydreaming and mind wandering to TUT bring out potential issues with the validity of self-report. The extent to which people can make discriminations between different forms of mind wandering and daydreaming is unclear. This lack of clarity is exacerbated in light of the fact that people are not always meta-aware of their mind wandering (Zedelius, Broadway, \& Schooler, 2015). This means that participants are reporting on experiences they are not aware of having until after the fact. While we think there are serious problems with thought probe methodologies, some of these problems can be mitigated by using probes in appropriate contexts. If anything, the present discussion suggests that thought probes might not be well-suited to discriminating between daydreaming and TUT, but this does not imply that thought probes cannot be used to classify thoughts on the basis of relatively simple, introspectible qualities. For example, in our sample thought probes presented in Figure 4, we ask participants about the content of their thought and the direction of focus. We think that these probes deliver reliable data even if they do not facilitate more fine-grained classifications.

A related complication is that researchers have tended to assume that trait-level measures of "mind wandering" necessarily index TUT. For instance, although commonly used trait-level measures of "mind wandering" (i.e., the mind wandering: spontaneous questionnaire and the 
mind wandering: deliberate questionnaire, Carriere et al., 2013) do not require the presence of a task for one to report "mind wandering," some researchers of TUT (ourselves included) have theoretically integrated findings from studies employing these trait-level questionnaires with findings from laboratory studies that directly indexed TUT (e.g., Golchert et al., 2017; Seli, Risko, \& Smilek, 2016a). If, however, these trait-level reports of mind wandering sometimes reflect task-free thoughts (which seems a reasonable assumption), it could be conceptually and theoretically problematic to equate these task-free reports with reports of TUT. This, in a way, is an extension of the methodological critique of mind wandering research raised in Seli, Kane, et al., 2018, although we here extend this critique by specifically applying it to the TUT variety of mind wandering and by offering some suggestions for a way forward.

We can observe the consequences of these methodological issues in studies that examine trait-level mind wandering. Godwin and colleagues (2017) found significant positive correlations between trait mind wandering and increased functional connectivity within the default mode network. However, functional connectivity was measured during resting state, which is a taskfree scenario. Trait mind wandering was assessed using items that measure the propensity for TUT (derived from Mrazek et al., 2013). Despite this mismatch in indices of "mind wandering," the fact that trait mind wandering was found to correlate with default mode activity in task free conditions is in some ways all the more remarkable, as there is no obvious connection between the trait-level variable and the corresponding default mode connectivity in a task-free scenario.

In other studies, research led by Gil-Jardiné and Mason identified significant positive correlations between trait mind wandering and both distracted thoughts while driving (GilJardiné et al., 2017) and having stimulus-independent thoughts while performing a habitual routine (Mason et al., 2007). While both measured TUT at the state level, each group used items 
from the daydreaming frequency scale (DDFS; Giambra, 1993) to determine trait mind wandering. Hence, neither shows a correlation between the propensity for TUT and state-level TUT. This is problematic because other researchers assume that the trait mind wandering measures in these studies are indexed to TUT. In fact, Fell (2018) and O'Callaghan et al. (2019) both cite Mason et al. (2007), despite the fact that the former are both interested in TUT.

As noted above, some trait-level measures of mind wandering do capture one's propensity to engage in TUT. Specifically, the mind wandering questionnaire (MWQ) developed by Mrazek et al. (2013) includes 5 items, each of which specifies the presence of a task in assessing mind wandering. These items include: "I have difficulty maintaining focus on simple or repetitive work" and "I do things without paying full attention." For researchers interested in TUT and trait-level measures of TUT, this scale is best in virtue of being precise about the tendency to be inattentive to a task. One lingering issue, however, is that the MWQ does not distinguish between the varieties of TUT outlined above. For example, if people report difficulty maintaining focus on simple or repetitive tasks, are they thinking in terms of other-imposed activities, self-selected activities, or a combination of the two? Again, when people report doing things without paying full attention, are they reporting repeated failure to attend adequately or that they do not devote full attention to everything they do? As we argued in Section 2, most daily-life tasks are such that people need not think very hard about doing them in order to perform the tasks successfully. Hence, the MWQ could be refined in light of our earlier methodological criticisms.

Moving forward, then, we recommend that researchers take caution in theoretically integrating results across studies that specifically index TUT and those that index task-free thought (or those in which it is unclear whether the thoughts in question are indeed task- 
unrelated). Moreover, researchers would benefit from the development of a trait-level measure that specifically indexes the different varieties of TUT outlined here (i.e., unintentional, undirected, successfully directed, and unsuccessfully directed). With the development of such a questionnaire, researchers can ensure that they are indexing the specific cognitive process of interest rather than potentially confounding this process with other, separable varieties of mind wandering (e.g., task-free thought) or TUT.

\section{Summary and Concluding Remarks}

The increased scientific interest in mind wandering makes this an exciting time to study the wandering mind. Increased interest should generate increased scrutiny, and there are still various methodological and conceptual challenges that need to be faced in order to develop more mature scientific theories of mind wandering. We have outlined three complications in investigations of the TUT variety of mind wandering, while highlighting the different ways in which these complications may be hindering the development of a scientific account of TUT. These complications include (a) the ambiguity of task-unrelatedness, which poses problems for classifying thoughts as 'task-unrelated', (b) ambiguities in the prompts used for daily-life studies of mind wandering, which make it difficult to compare daily-life measures of mind wandering can be directly compared to laboratory-based measures of TUT, and (c) the tendency for researchers to treat measures of mind wandering and daydreaming as exclusive indices of TUT, which also complicates trait-level measures of TUT.

Many of these methodological issues arise from the ambiguity of what constitutes a task. People engage in a variety of tasks, not all of which are focal, and daily life contains many taskfree and task-ambiguous scenarios. Further, the possibility of mind wandering in these scenarios suggests a distinction between mind wandering and TUT, which (as we noted) has several 
implications for current research into both mind wandering and TUT. Thus, attending to the rich variety of tasks that humans perform (and the way they go about performing them) should contribute to a more nuanced scientific account of TUT.

While these are complicated issues, we propose the following concrete suggestions to help guide future investigations of TUT. First, we suggest that researchers increase efforts to distinguish between the various kinds of TUT outlined in Section 1. The recently proposed distinction between intentional and unintentional mind wandering represents a first step, but, as we argued, there is room for greater nuance. Second, researchers should use caution when directly comparing results from daily life studies of TUT and laboratory-based studies of TUT. In particular, research should establish the kinds of tasks being performed across both studies (i.e., fluid or steady). Part of the fix can come from the study of daily life, which can ask questions to determine what kinds of tasks people are performing and how people view the attentional demands of such tasks. Third, researchers should use caution when theoretically integrating findings across different studies of mind wandering given that, as this article has revealed, not all measures of mind wandering consist in TUT. While this point was raised first in Seli, Kane, et al., 2018, we here proposed a scale that can be used to measure trait mind wandering that more accurately measures the propensity for TUT. Fourth, investigations of TUT should utilize more ecologically valid tasks that reflect the differences between sustained task performance and more fluid forms of goal pursuit. This, in turn, would allow for more appropriate comparisons between daily-life and lab-based TUT. This can be accomplished in two ways: (a) researchers might employ tasks with higher degrees of temporal fluidity, and (b) researchers might consider utilizing tasks that do not constantly place demands on participants' attention, which should in turn permit participants to engage in strategic TUT (e.g., Seli, 
Carriere, et al., 2018). These courses of action should provide more structured grounds for research on TUT, and in doing so, allow for the development of a more mature scientific account of the phenomenon. 


\section{References}

Baird, B., Smallwood, J., Mrazek, M. D., Kam, J. W., Franklin, M. S., \& Schooler, J. W. (2012). Inspired by distraction: mind wandering facilitates creative incubation. Psychological science, 23(10), 1117-1122.

Baird, B., Smallwood, J., \& Schooler, J. W. (2010, April). I can shake that feeling: Positive mind-wandering prevents the deterioration of mood. Poster presented at Towards a Science of Consciousness conference, Tucson, AZ.

Birnie, L.H., Smallwood, J., Reay, J., and Riby, L.M. 2015. "Glucose and the wandering mind: not paying attention or simply out of fuel?" Psychopharmacology 232:16, 2903-10.

Blanchard, N., Bixler, R., Joyce, T., \& D’Mello, S. (2014). Automated physiological-based detection of mind-wandering during learning. In S. Trausan-Matu, K. Boyer, M. Crosby, \& K. Panourgia (Eds.), Lecture Notes in Computer Science: Vol. 8474. Intelligent tutoring systems (pp. 55-60). New York, NY: Springer Science+Business Media. doi:10.1007/978-3-319-07221-0_7.

Buckner, R. L., Andrews-Hanna, J. R., \& Schacter, D. L. (2008). The brain's default network. Annals of the New York Academy of Sciences, 1124, 1-38.

Callard, F., Smallwood, J., Golchert, J., \& Margulies, D. S. (2013). The era of the wandering mind? Twenty-first century research on self-generated mental activity. Frontiers in Psychology, 4, 891.

Carciofo, R., Du, F., Song, N., \& Zhang, K. (2014). Mind-wandering, sleep quality, affect, and chronotype: An exploratory study. PLoS ONE, 9(3), Article e91285. doi:10.1371/journal.pone.0091285 
Carriere, J. S., Seli, P., \& Smilek, D. (2013). Wandering in both mind and body: Individual differences in mind wandering and inattention predict fidgeting. Canadian Journal of Experimental Psychology/Revue canadienne de psychologie expérimentale, 67(1), 19-31.

Casner, S. M., \& Schooler, J. W. (2013). Thoughts in Flight Automation Use and Pilots' TaskRelated and Task-Unrelated Thought. Human Factors: The Journal of the Human Factors and Ergonomics Society, 0018720813501550.

Christoff, K., Gordon, A. M., Smallwood, J., Smith, R., \& Schooler, J. W. (2009). Experience sampling during fMRI reveals default network and executive system contributions to mind wandering. Proceedings of the National Academy of Sciences, 106, 8719-8724.

Csikszentmihalyi, M., \& Larson, R. (1987). Validity and reliability of the experience sampling method: Mental disorders in their natural settings. Journal of Nervous and Mental Disease, 175, 526-536.

De Vries, M. C., Dijkman-Caes, C., \& Delespaul, P. (1990). The sampling of experience: A method of measuring the co-occurrence of anxiety and depression in daily life. In J. D. Maser \& C. R. Cloninger (Eds.), Comorbidity of mood and anxiety disorders (pp. 707726). Washington, DC: American Psychiatric Press.

Fell J. (2018). Is the Hippocampus a Potential Target for the Modulation of Mind Wandering in Major Depression?. Frontiers in psychiatry, 9, 363. doi:10.3389/fpsyt.2018.00363.

Feng, S., D’Mello, S., \& Graesser, A. C. (2013). Mind wandering while reading easy and difficult texts. Psychonomic bulletin \& review, 20(3), 586-592.

Franklin, M. S., Broadway, J. M., Mrazek, M. D., Smallwood, J., \& Schooler, J. W. (2013). Window to the wandering mind: Pupillometry of spontaneous thought while reading. The Quarterly Journal of Experimental Psychology, 66, 2289-2294. 
Gargiulo, S. (2013, October 30). Daydream believer: Is a wandering mind a creative mind? CNN Thinking Business. http://www.cnn.com/2013/10/30/business/daydream-believeris-a-wandering/

Giambra, L. M. (1989). Task-unrelated thought frequency as a function of age: a laboratory study. Psychology and Aging, 4, 136-143.

Giambra, L. M. (1993). The influence of aging on spontaneous shifts of attention from external stimuli to the contents of consciousness. Experimental gerontology, 28(4-5), 485-492.

Gil-Jardiné, C., Née, M., Lagarde, E., Schooler, J., Contrand, B., Orriols, L., and Galera, C. 2017. The distracted mind on the wheel: Overall propensity to mind wander is associated with road crash responsibility, PLoS ONE 12:8: e0181327

Godwin, C.A., Hunter, M.A., Bezdek, M.A., Lieberman, G., Elkin-Frankston, S., Romero, V.L., Witkiewitz, K., Clark, V.P., and Schumacher, E.H. 2017. "Functional connectivity within and between intrinsic brain networks correlates with trait mind wandering," Neuropsychologia 103, 140-53.

Golchert, J., Smallwood, J., Jefferies, E., Seli, P., Huntenburg, J. M., Liem, F., ... \& Margulies, D. S. (2017). Individual variation in intentionality in the mind-wandering state is reflected in the integration of the default-mode, fronto-parietal, and limbic networks. Neuroimage, 146, 226-235.

Hulburt, R. T. (1997). Randomly sampling thinking in the natural environment. Journal of Consulting and Clinical Psychology, 65, 941-944.

Irving, Z. C. (2016). Mind-wandering is unguided attention: Accounting for the "purposeful" wanderer. Philosophical Studies, 173, 547-571. 
Kane, M. J., Brown, L. H., McVay, J. C., Silvia, P. J., Myin-Germeys, I., \& Kwapil, T. R. (2007). For whom the mind wanders, and when: An experience-sampling study of working memory and executive control in daily life. Psychological science, 18(7), 614621.

Kane, M. J., Gross, G. M., Chun, C. A., Smeekens, B. A., Meier, M. E., Silvia, P. J., \& Kwapil, T. R. (2018). For whom the mind wanders, and when, varies across laboratory and dailylife settings. Psychological science, 28(9), 1271-1289.

Kane, M. J., \& McVay, J. C. (2012). What mind wandering reveals about executive-control abilities and failures. Current Directions in Psychological Science, 21(5), 348-354.

Killingsworth, M. A., \& Gilbert, D. T. (2010). A wandering mind is an unhappy mind. Science, $330(6006), 932$.

Klinger, E. (1971). Structure and functions of fantasy (New York: Wiley).

Klinger, E. (1978-79). Dimensions of thought and imagery in normal waking states. Journal of Altered States of Consciousness, 4, 97-113.

Klinger, E. (2013). Goal commitments and the content of thoughts and dreams: Basic principles. Frontiers in psychology 4, doi: 10.3389/fpsyg.2013.00415.

Klinger, E. (2018). The goal theory of current concerns and its applications at year 45. In N. Baumann, M. Kazén, M. Quirin, \& S. Koole (eds.), Why people do the things they do: Building on Julius Kuhl's Contributions to the Psychology of Motivation and Volition (Göttingen: Hogrefe), 33-54.

Klinger, E., \& Cox, W. M. (1987-88). Dimensions of thought flow in everyday life. Imagination, Cognition and Personality, 7, 105-128. 
Klinger, E., \& Cox, W.M. (2011). Motvation and the goal theory of current concerns. In W.M. Cox \& E. Klinger (eds.), Handbook of motivational counselling ( $2^{\text {nd }}$ ed.) (Chichester, UK: Wiley), 3-47.

Klinger, E., Marchetti, I., \& Koster, E.H. W. (2018). Spontaneous thought and goal pursuit: From functions such as planning to dysfunctions such as rumination, In K. Fox \& K. Christoff (eds.) The Oxford Handbook of Spontaneous Thought (Oxford: Oxford University Press).

Krasich, K., McManus, R., Hutt, S., Faber, M., D’Mello, S.K., and Brockmole, J.R. (2018). Gaze-based Signatures of Mind Wandering During Real-World Scene Processing. Journal of Experimental Psychology: General, 147:8, 1111-24.

Mason, M.F., Norton, M.I., Van Horn, J.D., Wegner, D.M., Grafton, S.T., and Macrae, C.N. (2007). Wandering Minds: The Default Network and Stimulus-Independent Thought. Science 315:5810, 393-95.

McVay, J. C., \& Kane, M. J. (2009). Conducting the train of thought: working memory capacity, goal neglect, and mind wandering in an executive-control task. Journal of Experimental Psychology: Learning, Memory, and Cognition, 35, 196-204.

McVay, J. C., \& Kane, M. J. (2010). Does mind wandering reflect executive function or executive failure? Comment on Smallwood and Schooler (2006) and Watkins (2008). Psychological Bulletin, 136, 188-197.

McVay, J. C., Kane, M. J., \& Kwapil, T. R. (2009). Tracking the train of thought from the laboratory into everyday life: An experience-sampling study of mind wandering across controlled and ecological contexts. Psychonomic bulletin \& review, 16(5), 857-863. 
Meier, M. E. (2018). Can research participants comment authoritatively on the validity of their self-reports of mind wandering and task engagement? A replication and extension of Seli, Jonker, Cheyne, Cortes, and Smilek (2015). Journal of Experimental Psychology: Human Perception and Performance, 44(10), 1567-1585.

Mills, C., Raffaelli, Q., Irving, Z. C., Stan, D., \& Christoff, K. (2018). Is an off-task mind a freely-moving mind? Examining the relationship between different dimensions of thought. Consciousness and cognition 58, 20-33 doi:

https://doi.org/10.1016/j.concog.2017.10.003

Mooneyham, B. W., \& Schooler, J. W. (2013). The costs and benefits of mind-wandering: a review. Canadian Journal of Experimental Psychology/Revue canadienne de psychologie expérimentale, 67(1), 11-18.

Mrazek, M. D., Phillips, D. T., Franklin, M. S., Broadway, J. M., \& Schooler, J. W. (2013). Young and restless: Validation of the mind-wandering questionnaire (MWQ) reveals disruptive impact of mind-wandering for youth. Frontiers in Psychology, 4, 7.

O’Callaghan, C., Shine, J.M., Hodges, J.R., Andrews-Hanna, J.R., and Irish, M. (2019). "Hippocampal atrophy and intrinsic brain network dysfunction relate to alterations in mind wandering in neurodegeneration," Proceedings of the National Academy of Sciences USA 116:8, 3316-21.

Qin, J. et al. 2011. "Dissociation of Subjectively Reported and Behaviorally Indexed Mind Wandering by EEG Rhythmic Activity," PLoS One 6:9: e23124

Qu, W., Ge, Y., Xiong, Y., Carciofo, R., Zhao, W., \& Zhang, K. (2015). The relationship between mind-wandering and dangerous driving behavior among Chinese drivers. Safety Science, 78, 41-48. 
Raichle, M.E. (2015). The brain's default mode network. Annual Review of Neuroscience, 38, 433-447.

Robison, M.K. \& Unsworth, N. 2018. Cognitive and contextual correlates of spontaneous and deliberate mind wandering. Journal of Experimental Psychology: Learngin, Memory, and Cognition 44:1, 85-98.

Rummel, J., \& Boywitt, C. D. (2014). Controlling the stream of thought: Working memory capacity predicts adjustment of mind-wandering to situational demands. Psychonomic Bulletin \& Review, 21, 1309-1315.

Sapolsky, R. M. (2015, June 19). The benefits of mind-wandering. The Wall Street Journal. http://www.wsj.com/articles/the-benefits-of-mind-wandering-1434716243

Schad, D. J., Nuthmann, A., \& Engbert, R. (2012). Your mind wanders weakly, your mind wanders deeply: objective measures reveal mindless reading at different levels. Cognition, 125(2), 179-194.

Schooler, J. W. (2002). Re-representing consciousness: Dissociations between experience and meta-consciousness. Trends in Cognitive Sciences, 6, 339-344.

Schooler, J. W., Reichle, E. D., \& Halpern, D. V. (2004). Zoning out while reading: Evidence for dissociations between experience and metaconsciousness. In D. T. Levin (Ed.), Thinking and seeing: Visual metacognition in adults and children (pp. 203-226). Cambridge, MA: MIT Press.

Seli, P. (2016). The Attention-Lapse and Motor Decoupling accounts of SART performance are not mutually exclusive. Consciousness and Cognition, 41, 189-198.

Seli, P., Beatty, R.E., Cheyne, J.A., Smilek, D., Oakman, J., Schacter, D.L. (2018). How Pervasive is Mind Wandering, Really? Consciousness and Cognition 66, 74-78. 
Seli, P., Carriere, J. S., \& Smilek, D. (2015). Not all mind wandering is created equal:

Dissociating deliberate from spontaneous mind wandering. Psychological Research, 79(5), 750-758.

Seli, P., Carriere, J. S., Thomson, D. R., Cheyne, J. A., Martens, K. A. E., \& Smilek, D. (2014). Restless mind, restless body. Journal of Experimental Psychology: Learning, Memory, and Cognition, 40(3), 660-668.

Seli, P., Carriere, J. S., Wammes, J. D., Risko, E. F., Schacter., D. L., \& Smilek, D. (2018). On the clock: Evidence for the rapid and strategic modulation of mind-wandering. Psychological Science.

Seli, P., Cheyne, J. A., \& Smilek, D. (2013). Wandering minds and wavering rhythms: Linking mind wandering and behavioral variability. Journal of Experimental Psychology: Human Perception and Performance, 39, 1-5.

Seli, P., Cheyne, J. A., Xu, M., Purdon, C., \& Smilek, D. (2015). Motivation, intentionality, and mind wandering: Implications for assessments of task-unrelated thought. Journal of Experimental Psychology: Learning, Memory, and Cognition, 41, 1417-1425.

Seli, P., Jonker, T. R., Cheyne, J. A., Cortes, K., \& Smilek, D. (2015). Can research participants comment authoritatively on the validity of their self-reports of mind wandering and task engagement?. Journal of Experimental Psychology: Human Perception and Performance, 41, 703-709.

Seli, P., Kane, M. J., Metzinger, T., Smallwood, J., Schacter, D. L., Maillet, D., ... \& Smilek, D. (2018). The family-resemblances framework for mind-wandering remains well clad. Trends in cognitive sciences, 22(11), 959-961. 
Seli, P., Kane, M. J., Smallwood, J., Schacter, D. L., Maillet, D., Schooler, J. W., \& Smilek, D. (2018). Mind-wandering as a natural kind: A family-resemblances view. Trends in cognitive sciences, 22(6), 479-490.

Seli, P., Risko, E. F. R., Purdon, C., \& Smilek, D. (2016). Intrusive thoughts: Linking spontaneous mind wandering and OCD symptomatology. Psychological Research. doi: DOI: $10.1007 / \mathrm{s} 00426-016-0756-3$

Seli, P., Risko, E. F., \& Smilek, D. (2016a). Assessing the associations among trait and state levels of deliberate and spontaneous mind wandering. Consciousness and Cognition, 41, $50-56$.

Seli, P., Risko, E. F., \& Smilek, D. (2016b). On the necessity of distinguishing between unintentional and intentional mind wandering. Psychological science, 27(5), 685-691.

Seli, P., Risko, E. F., Smilek, D., \& Schacter, D. L. (2016). Mind-wandering with and without intention. Trends in Cognitive Sciences, 20(8), 605-617.

Seli, P., Smallwood, J., Cheyne, J. A., \& Smilek, D. (2015). On the relation of mind-wandering and ADHD symptomatology. Psychonomic Bulletin \& Review, 22, 629-636. doi:10.3758/s13423-014-0793-0

Seli, P., Smallwood, J., Kane, M. J., Schacter, D. L., Maillet, D., Schooler, J. W., \& Smilek, D. (2018). Mind-wandering as a natural kind: A family-resemblances view. Trends in Cognitive Sciences, 22(6), 479-490.

Seli, P., Wammes, J. D., Risko, E. F., \& Smilek, D. (2015). On the relation between motivation and retention in educational contexts: The role of intentional and unintentional mindwandering. Psychonomic Bulletin \& Review. Advance online publication. doi:10.3758/s13423-015-0979-0 
Shrimpton, D. et al. 2017. "Daydream Believer: Rumination, Self-Reflection and the Temporal Focus of Mind Wandering Content," Europe's Journal of Psychology 13:4, 784-809.

Singer, J. L., \& Antrobus, J. S. (1972). Daydreaming, imaginal processes, and personality: A normative study. The function and nature of imagery, 175-202.

Smallwood, J. \& Andrews-Hanna, J. (2013). Not all minds that wander are lost: The importance of a balanced perspective on the mind wandering state. Frontiers in Psychology 4, 441.

Smallwood, J., Beach, E., Schooler, J. W., \& Handy, T. C. (2008). Going AWOL in the brain: Mind wandering reduces cortical analysis of external events. Journal of Cognitive Neuroscience, 20, 458-469.

Smallwood, J., Karapanagiotidis, T., Ruby, F., Medea, B., de Caso, I., Konishi, M., ... \& Jefferies, E. (2016). Representing Representation: Integration between the Temporal Lobe and the Posterior Cingulate Influences the Content and Form of Spontaneous Thought. PloS One, 11(4), e0152272.

Smallwood, J., McSpadden, M., \& Schooler, J. W. (2007). The lights are on but no one's home: Meta-awareness and the decoupling of attention when the mind wanders. Psychonomic Bulletin \& Review, 14, 527-533.

Smallwood, J., Nind, L., \& O'Connor, R. C. (2009). When is your head at? An exploration of the factors associated with the temporal focus of the wandering mind. Consciousness and Cognition, 18, 118-125.

Smallwood, J., \& Schooler, J. W. (2006). The restless mind. Psychological Bulletin, 132, 946958.

Smallwood, J., \& Schooler, J. W. (2015). The science of mind wandering: empirically navigating the stream of consciousness. Annual Review of Psychology, 66, 487-518. 
Smith, A., Murray, S., Schooler, J., and Seli, P. In Preparation. Comparing Dichotomous and Continuous Measures of Mind Wandering on Task Performance.

Song, X., \& Wang, X. (2012). Mind wandering in Chinese daily lives-an experience sampling study. PloS One, 7(9), e44423.

Stawarczyk, D., Majerus, S., Van der Linden, M., \& D'Argembeau, A. (2012). Using the daydreaming frequency scale to investigate the relationships between mind-wandering, psychological well-being, and present-moment awareness. Frontiers in psychology, 3, 363.

Thomson, D. R., Besner, D., \& Smilek, D. (2015). A resource-control account of sustained attention: Evidence from mind-wandering and vigilance paradigms. Perspectives on Psychological Science, 10, 82-96.

Wammes, J. D., Boucher, P. O., Seli, P., Cheyne, J. A., \& Smilek, D. (2013). Mind wandering during lectures I: Changes in rates across an entire semester. Scholarship of Teaching and Learning in Psychology, 2(1), 13-32.

Wammes, J. D., Seli, P., Cheyne, J. A., Boucher, P. O., \& Smilek, D. (2016). Mind wandering during lectures II: Relation to academic performance. Scholarship of Teaching and Learning in Psychology, 2(1), 33-48.

Weinstein, Y. (2018). Mind-wandering, how do I measure thee with probes? Let me count the ways. Behavior research methods 50:2, 642-61.

Yanko, M. R., \& Spalek, T. M. (2013). Route familiarity breeds inattention: A driving simulator study. Accident Analysis \& Prevention, 57, 80-86.

Yanko, M. R., \& Spalek, T. M. (2014). Driving with the wandering mind: the effect that mindwandering has on driving performance. Human factors, 56(2), 260-269. 
Zedelius, C. M., \& Schooler, J. W. (2015). The Richness of Inner Experience: Relating Styles of Daydreaming to Creative Processes. Frontiers in psychology, 6, 2063.

doi:10.3389/fpsyg.2015.02063 